\title{
Control de escorrentías urbanas mediante Sistemas Urbanos de Drenaje Sostenible (SUDS): Pozos/Zanjas de infiltración ${ }^{1}$
}

\section{Urban runoff control through Sustainable Urban Drainage Systems (SUDS): Wells / Infiltration trenches}

\section{Controlar escoamento urbano através de Sistemas de Drenagem Urbano Sustentável (SUDS): Poços / Trincheiras de infiltração}

\author{
E. D. Cubides y G. E. Santos
}

Recibido: julio 19 de 2017 - Aceptado: junio 28 de 2018

\begin{abstract}
Resumen- En el marco del manejo eficiente del recurso hídrico y el medio ambiente, el control de las escorrentías superficiales es un área de estudio de gran importancia en el desarrollo de las ciudades, pues su crecimiento exponencial y el aumento de zonas duras no permeables hace que el transporte de la escorrentía tome tiempos mayores. Consecuentemente, se generan implicaciones ambientales y económicas importantes que conllevan a la implementación de estructuras que den mayor holgura para el respectivo transporte. Cuando no es posible el control mediante estos elementos, se presentan fenómenos de inundación que afectan la calidad de vida de la población $y$ se deben generar planes de emergencia para mitigar estos riesgos. La implementación de sistemas de drenaje sostenible
\end{abstract}

${ }^{1}$ Producto derivado del proyecto de grado de maestría "Control de escorrentías urbanas mediante pozos de infiltración: Aplicación práctica a un tramo del Colector de aguas lluvias de la localidad de Fontibón, Bogotá, D.C.", presentado por el aspirante a maestría Elkin D. Cubides C. y dirigido por G. E., Santos., de la Escuela Colombiana de Ingeniería "Julio Garavito".

E.D., Cubides, Consultor privado, email: duvan.cubides@hotmail. com.

G. E., Santos, Escuela Colombiana de Ingeniería "Julio-Garavito", Bogotá, Colombia, email: german.santos@escuelaing.edu.co.

Como citar este artículo: Cubides, E. D. y Santos, G. E. Control de escorrentías urbanas mediante Sistemas Urbanos de Drenaje Sostenible (SUDS): Pozos/Zanjas de infiltración, Entre Ciencia e Ingeniería, vol. 12, no. 24, pp. 32-42, julio-diciembre, 2018.

DOI: http://dx.doi.org/10.31908/19098367.3813

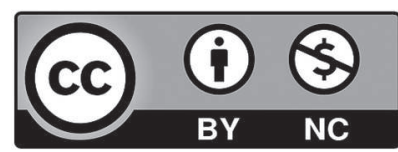

Attribution-NonCommercial 4.0 International (CC BY-NC 4.0) alternativos como los Sistemas de Drenaje Urbano Sostenibles (SUDS), permite aminorar y retrasar los picos de tormenta que se presentan en las ciudades. A continuación, se presenta un análisis de resultados correspondiente a la respuesta analítica mediante la modelación hidráulica e hidrológica y la aplicación de EPA-SWMM, programa desarrollado por la U.S. Environmental Protection Agency (EPA) para el análisis de lluvia y comportamiento hidráulico de redes de alcantarillado.

Palabras clave - SUDS, Hidrología, Hidráulica, Pozos/ Zanjas de infiltración, EPA SWMM, Controles LID, SaintVenant, BMPs.

Abstract - In the framework of the efficient management of water resources and the environment, the control of the surface runoff is an area of study of great importance in the development of cities, because their exponential growth and the increase of non-permeable hard zones produces greater runoff transport times. Thus, environmental and economic implications are generated that leads to the implementation of mayor structures for the respective transport. When the control by these elements is not possible, flood phenomena affect the quality of life of the population and emergency plans for these risks must be generated. The implementation of alternative sustainable drainage systems such as Sustainable Urban Drainage Systems (SUDS) allows to reduce and to delay the storm surges that occur in. Next, an analysis of results related to hydraulic and hydrological modeling and the EPA-SWMM, a program developed by the United States Environmental Protection Agency (EPA) for the analysis of rainfall and hydraulic behavior of networks of Sewerage

Keywords - SUDS, Hydrology, Hydraulics, infiltration trench, EPA SWMMM, LID Controls, Saint-Venant, BMPs.

Resumo - Sob a gestão eficiente dos recursos hídricos e do meio ambiente, controlando o escoamento superficial é uma área de estudo de grande importância para o desenvolvimento das cidades, porque devido ao crescimento exponencial destes 
e aumento de áreas de difícil não permeáveis transporte leva a gerar um escoamento envolvendo tempos mais longos; assim, implicações ambientais e económicas significativas que afetam a implementação de estruturas de maior folga para o respectivo transporte e consideravelmente afeta o uso do solo na instalação do mesmo são gerados. Quando não é possível controlar por esses elementos, inundações ocorrem afetando a qualidade de vida da população e deve gerar planos de contingência para mitigar esses riscos. Sistemas alternativos, tais como sistemas de drenagem sustentáveis drenagem sustentável Urbana (SUDS) permitir a sua aplicação reduzir pico tempestade ocorrendo em cidades de acordo com o volume, em que são aplicados esses sistemas. Em seguida, uma análise correspondente para os resultados analíticos pela resposta hidráulica e modelagem hídrico e aplicação do programa de EPA-SWMM desenvolvidos por os EUA apresentado Agência de Proteção Ambiental (EPA) para analisar o comportamento hidráulico das redes de saneamento.

Palavras chave - SUDS, Hidrologia, Hidraulica, poços / trincheiras de infiltração, EPA SWMM, Controles LID, SaintVenant, BMPs.

\section{NOMENCLATURA}

SUDS: acrónimo de Sistema de Drenaje Urbano Sostenible.

EPA-SWMM: programa desarrollado por la EPA para la planeación, análisis y diseño de sistemas de alcantarillado de aguas lluvias, combinados y sanitarios y otros sistemas de drenaje urbano. Las siglas SWMM corresponden a Storm Water Management Model.

HIDROGRAMA: el hidrograma es un gráfico que presenta el comportamiento en el tiempo de una condición hidrológica.

HIETOGRAMA: el hietograma especifica la profundidad de precipitación que ocurre en $\mathrm{n}$ intervalos de tiempo sucesivos de duración $\Delta \mathrm{t}$ sobre una duración total de $\mathrm{T}=\mathrm{n}$ $\Delta \mathrm{T}$.

CONTROLES LID: son controles de modelación diseñados para el uso del programa desarrollado por la EPA; SWMM v5.1, los cuales implementan una alternativa de drenaje urbano con el fin de mejorar la calidad de agua y disminuir el aporte generado a los colectores de aguas lluvias proyectados. La sigla LID corresponde a Low Impact Development Control Units.

\section{INTRODUCCIÓN}

A través del tiempo, el estudio del ciclo hidrológico y las inundaciones que se generan especialmente en zonas urbanas, ha sido un tema de investigación importante, pues la atenuación de estos eventos o tormentas en los sistemas de drenaje convencionales existentes es insuficiente. El aumento de la población de manera exponencial hace aún más complicada una buena gestión en este aspecto $\mathrm{y}$, por tanto, se convierte en un reto para la ingeniería.

En distintas ciudades de Colombia es claro que se necesita la implementación de nuevos sistemas que no reemplacen, pero sí complementen, los sistemas convencionales para mejorar cada uno de los procesos que se presentan en la gestión de las escorrentías. De acuerdo con [1], el desarrollo económico, a pesar de los grandes esfuerzos realizados para fortalecer los procesos de planificación y protección de los componentes ecológicos, ha generado que la intervención sobre el territorio haya estado acompañada de acciones como la desecación de humedales y ciénagas, la pérdida de bosques y cobertura vegetal, con las consecuencias que esto tiene en términos de erosión y aumento de escorrentías, afectando de manera importante las fuentes de agua y la estabilidad del terreno, haciéndolo susceptible a deslizamientos, inundaciones y avenidas torrenciales en zonas que antes no lo eran.

Por lo anterior, a nivel urbano, es necesario implementar nuevas tecnologías de drenaje. En este sentido, como alternativa de drenaje sostenible se presentan los pozos o zanjas de infiltración, los cuales además de aminorar los picos de tormenta ayudando a disminuir circunstancialmente el aporte de caudales al sistema de drenaje urbano convencional, también pueden considerarse como alternativas de tratamiento previo, antes de realizarse la gestión del agua lluvia y entregarse a un cuerpo de agua.

Teniendo en cuenta lo anterior, para analizar el uso de nuevas alternativas de drenaje complementadas con sistemas de drenaje urbano sostenibles, en el presente estudio se utilizó la herramienta computacional EPA SWMM en su versión 5.1. En este software libre de análisis elaborado, la EPA presenta un nuevo módulo de análisis hidrológico en [2] y [3], en el cual se adiciona un aparte correspondiente a los controles LID (Low Impact Development). Dentro de las opciones que presenta dicha herramienta se encuentra el sistema de drenaje sostenible SUDS, dando paso al siguiente análisis.

\section{Generalidades y GeSTión DEL AGUA}

Los problemas que hoy en día se viven en las ciudades de Colombia con respecto a la gestión del agua lluvia, implican realizar una búsqueda continua de alternativas que ayuden a que su manejo sea cada vez más eficiente, pues este problema de ingeniería tiene una gran connotación en la población que se beneficia.

Así, como complemento a los sistemas de drenaje urbano convencionales se han desarrollado nuevas tecnologías de captación, retención e infiltración, que permiten aminorar las cargas sobre estos sistemas y atenuar las tormentas de diseño predispuestas en él de una manera más amigable con el ambiente, siguiendo los patrones de la cuenca inalterada. Según [4], estas nuevas tecnologías hacen referencia a sistemas de drenaje urbano sostenible (SUDS), también conocidos mundialmente como:

- BMPs (Best Management Practices)

- MPC (Mejores Prácticas de Control)

- BPAs (Buenas Prácticas Ambientales)

- TEDUS (Técnicas de Drenaje Urbano Sostenible)

- LID (Low Impact Development)

- WSUD (Water Sensitive Urban Design)

- Diseño Urbano Sensible de Agua

Particularmente, en este trabajo se estudiaron los pozos/ 
zanjas de infiltración que son una alternativa de los SUDS, con la cual no solo se atenúan las tormentas de diseño previstas para el punto de interés, sino que funciona de manera combinada con los sistemas de drenaje convencionales y permite, al mismo tiempo, mejorar la calidad de agua de los vertidos de las redes de alcantarillado pluvial a los cuerpos de agua existentes.

\section{A. Planteamiento del problema}

Bogotá se localiza dentro de la zona de confluencia intertropical, la cual cruza la ciudad dos veces al año, situación que influye en el comportamiento de las lluvias produciendo dos épocas de lluvias que popularmente se denominan invierno. De acuerdo con [5], la primera se presenta en los meses de marzo, abril y mayo, y la segunda en los meses de septiembre, octubre y noviembre. Espacialmente hablando, la precipitación se caracteriza por valores medios que oscilan de 69 mm en la localidad de Bosa, al occidente, y los 142 $\mathbf{m m}$ en el sector de Torca, al norte de la ciudad.

Según [6], el incremento de la concentración de la población en las ciudades ha generado un aumento brutal de la urbanización de suelos y, por lo tanto, de su impermeabilidad, produciendo un mayor volumen de escorrentía y una elevación en las velocidades de los flujos naturales, reduciendo los tiempos de concentración, produciendo temperaturas más altas, caudales de base más bajos durante la época de estiaje y niveles de contaminación mayores. Estos efectos tienen consecuencias drásticas en cuanto a los volúmenes y los caudales punta o caudales pico, que se producen y se deben transportar en la red de alcantarillado.

Algunos de los ejemplos que se han vivido en los últimos años en la capital se muestran en las Fig. 1. - 2. - 3.:

En marzo 10 de 2008, la Avenida Caracas con Calle 26 se inundó. Este sector aparece identificado como una zona crítica por la Unidad de Emergencias, por ser un lugar con un alto porcentaje de basuras en su alcantarillado, lo que no permite evacuar rápidamente las aguas.

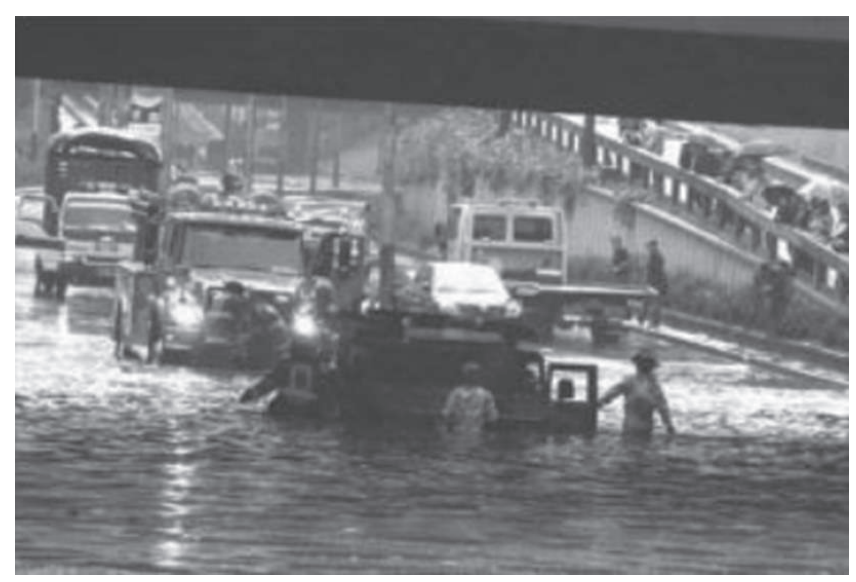

Fig. 1. Encharcamiento Av. Caracas con Calle 26. (www.elespectador.com)

Abril 2 de 2011, encharcamiento Carrera $7^{\mathrm{a}}$ con Calle 72.

Noviembre 20 de 2017, encharcamiento Autopista Norte con Calle 127.

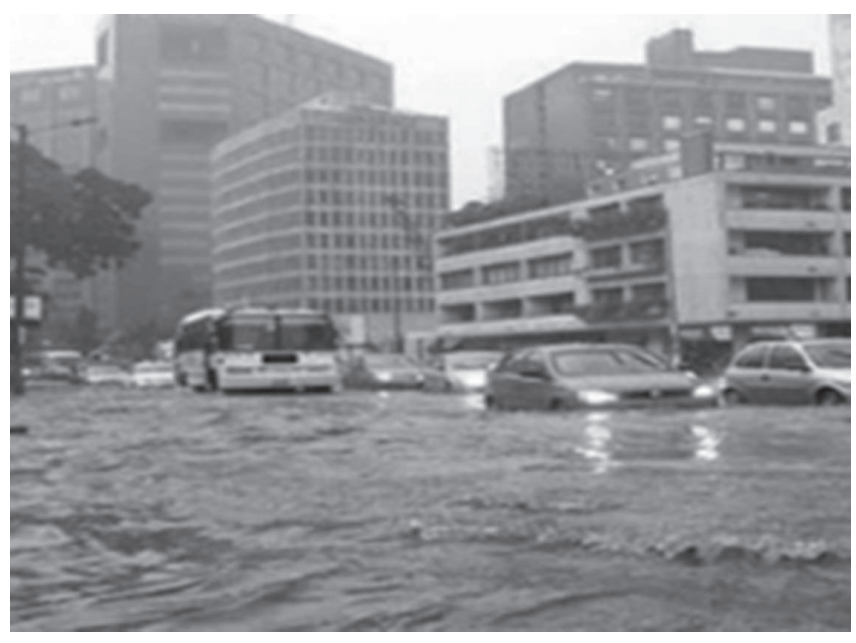

Fig. 2. Encharcamiento Carrera 7 con Calle 72. (www.elespectador.com)

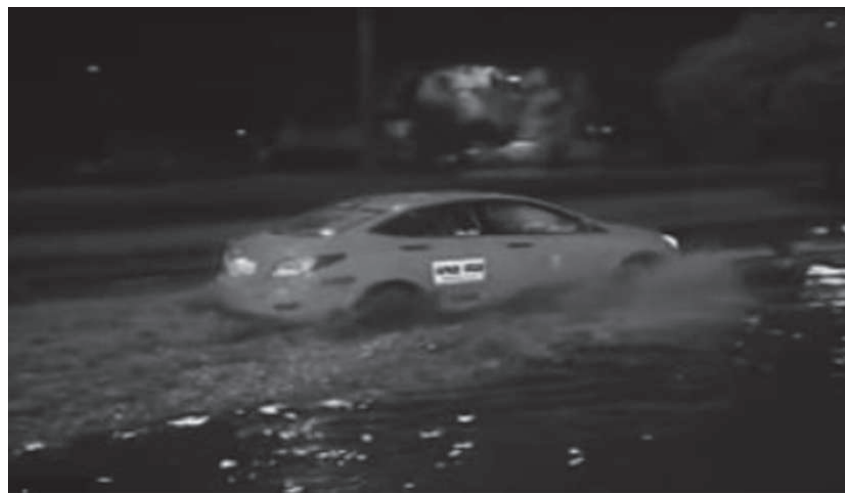

Fig. 3. Encharcamiento Autopista Norte con Calle 127. (www.eltiempo. com)

De acuerdo con lo anterior, la gestión de la escorrentía se convierte en una de las problemáticas más importantes que tiene la ciudad de Bogotá, pues la amortiguación de aguas lluvias se vuelve cada vez más complicada debido a que el rápido desarrollo urbano ha generado una gran impermeabilización de los suelos, dejando muy pocas coberturas vegetales que ayuden a interceptar el agua lluvia.

Es por esto que se presenta como complemento de los sistemas de drenaje urbano convencionales existentes, los SUDS, específicamente los pozos/zanjas de infiltración, los cuales permitirían aminorar, amortiguar y mejorar la calidad del vertido de los caudales picos de tormenta.

\section{B. Concepción del Proyecto y análisis técnico}

\section{Concepción del proyecto}

El caso de estudio utilizado se desarrolla en la localidad de Fontibón (Localidad 9), específicamente en el sector ubicado sobre las calles 13 y 17, entre la Av. Ciudad de Cali y la Carrera 96B, incluyendo la denominada "Ye" de Fontibón, con un área aproximada de estudio de 6.2 Ha. Entre los cursos de agua más importantes que se encuentran en inmediaciones de la zona de proyecto se encuentra el Río Fucha y el Canal Cundinamarca.

En la Fig. 4. se presenta la concepción y aplicación en el control y la gestión de la escorrentía en cuencas urbanas, 
concretamente, el proceso y desarrollo de la aplicación de los pozos/zanjas de infiltración en los sistemas de alcantarillado pluvial.

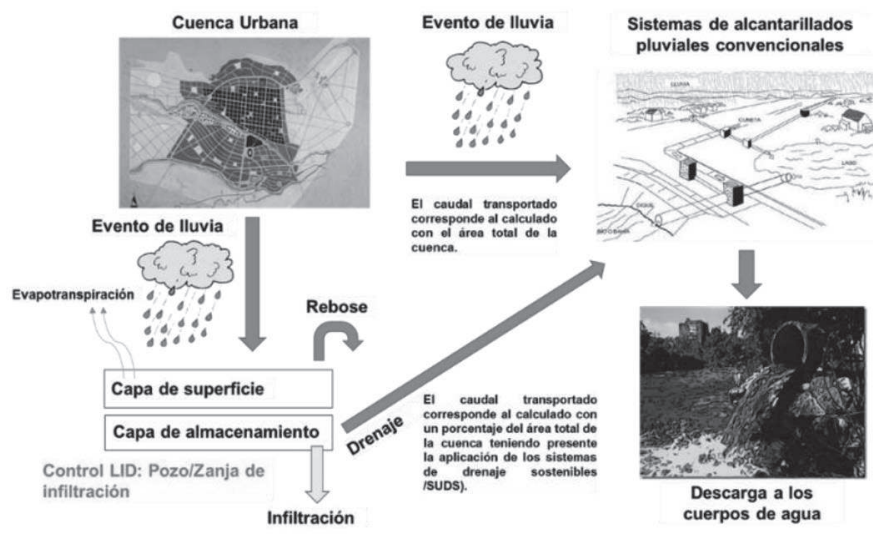

Fig. 4. Cadena de gestión de escorrentía de los sistemas de alcantarillado convencionales vs la aplicación de los sistemas SUDS (pozos/zanjas de infiltración).

Antes de implementar los pozos/zanjas de infiltración se deben contemplar aspectos relevantes en la etapa de diseño o prefactibilidad; según [7], debe tenerse en cuenta el tipo de terreno sobre el que se está trabajando, su tasa de infiltración debe ser adecuada y el nivel freático no debe verse afectado por la excavación. Además, no se pueden construir estos sistemas demasiado cerca de un edificio o una carretera para no afectar las cimentaciones.

De acuerdo con [8], se suelen emplear geotextiles de filtro y separación para envolver el material granular, y desagües de emergencia para, en el caso de que se supere la capacidad de depósito de diseño, poder enviar el sobrante a la red de alcantarillado. También se dispone una tubería permeable en la base que recoge el agua, lo que implica la construcción de pozos de registro para los cambios de dirección y la limpieza de la zanja.

\section{Análisis Hidrológico}

En el análisis hidrológico, debe contemplarse el análisis de lluvia, la metodología para el cálculo de caudales máximos y la metodología para el cálculo de hietogramas de diseño.

Para realizar el análisis de lluvia para el caso de drenaje urbano, es necesario caracterizar y recopilar información de la zona de estudio siguiendo estos pasos:

- Recopilar información de estaciones hidroclimatológicas (precipitación media multianual, mensual, humedad relativa, temperatura, evaporación y brillo solar, entre otros).

- Análisis de probabilidad de ocurrencia de tormentas máximas en 24 horas (Pmáx24horas).

- Elaboración de la Curva de Intensidad-DuraciónFrecuencia o mapa de intensidades para determinar el valor aproximado de la precipitación de la lluvia en el sitio de interés.

En las ciudades de Colombia, la entidad encargada del aseo y operación de los sistemas de acueducto y alcantarillado, en este caso la Empresa de Acueducto de Bogotá (EAB-ESP), dispone de una zonificación en la ciudad en la que, de acuerdo con las mediciones de precipitaciones previas, se obtuvo de los parámetros la curva IDF.

TABLA I

ParÁmetros hidrológicos - CURVA IDF - EAB-ESP

\begin{tabular}{|c|c|c|c|}
\hline TR & C1 & Xo & C2 \\
\hline $\mathbf{3}$ & 2336.26 & 18.1 & -1.02 \\
\hline $\mathbf{5}$ & 2558.88 & 17.5 & -1.01 \\
\hline $\mathbf{1 0}$ & 3261.18 & 18.7 & -1.03 \\
\hline
\end{tabular}

De la forma:

$\mathrm{I}=\mathrm{C}_{1} *\left(\mathrm{D}+\mathrm{X}_{\mathrm{o}}\right)^{\mathrm{C} 2}$

Donde:

I: Intensidad de la lluvia [mm/hr]

D: Duración de la lluvia [min]

$\mathrm{C}_{1}, \mathrm{X}_{\mathrm{o}} \mathrm{y}_{2}$ : Parámetros de la ecuación producto de un análisis de frecuencias, EAB-ESP (Bogotá).

Para el cálculo de caudales cuencas cuyo tamaño no supere $250 \mathrm{Ha}$, se aplicó el método racional, el cual calcula el caudal pico de aguas lluvias con base en la intensidad media del evento de precipitación, con una duración igual al tiempo de concentración del área de drenaje y un determinado coeficiente de escorrentía.

De acuerdo con el método racional, el caudal pico ocurre cuando toda el área de drenaje está contribuyendo, y este es una fracción de la precipitación media bajo las siguientes suposiciones: el caudal pico en cualquier punto es una función directa de la intensidad de la lluvia, durante el tiempo de concentración para ese punto. La frecuencia del caudal pico es la misma que la frecuencia media de la precipitación. El tiempo de concentración está implícito en la determinación de la intensidad media de la lluvia.

Es importante destacar que el tiempo de concentración seleccionado es el correspondiente al recomendado en estructuras de drenaje urbano, en este caso, en el que la entidad encargada de la operación de los sistemas de acueducto y alcantarillado indique.

Para obtener los hietogramas de diseño a partir de las curvas de Intensidad-Duración-Frecuencia (IDF), se optó por implementar la metodología del bloque alterno; según [9], el método del bloque alterno es una forma simple para desarrollar un hietograma de diseño utilizando una curva de intensidadduración-frecuencia. El hietograma de diseño producido por este método especifica la profundidad de precipitación que ocurre en $\mathrm{n}$ intervalos de tiempo sucesivos de duración $\Delta \mathrm{t}$ sobre una duración total de $\mathrm{Td}=\mathrm{n} \Delta \mathrm{T}$.

Después de seleccionar el periodo de retorno de diseño, la intensidad es leída en una curva IDF para cada una de las duraciones $\Delta \mathrm{t}, 2 \Delta \mathrm{t}, 3 \Delta \mathrm{t}, \ldots$, y la profundidad de precipitación correspondiente se encuentra al multiplicar la intensidad y la duración. Tomando diferencias entre valores sucesivos de profundidad de precipitación, se encuentra la cantidad de precipitación que debe añadirse por cada unidad adicional de 
tiempo $\Delta$ t. Estos incrementos o bloques se reordenan en una secuencia temporal, de modo que la intensidad máxima ocurra en el centro de la duración requerida $\mathrm{Td}$, y que los demás bloques queden en orden descendente alternativamente hacia la derecha y hacia la izquierda del bloque central, para formar el hietograma de diseño.

TABLA II

Cálculo del hietograma de diseño - Parámetros

\begin{tabular}{|c|c|}
\hline Tr_ & 3 años \\
\hline Precipitación aplicada [mm] & 28.44 \\
\hline \multicolumn{2}{|c|}{ Precipitación efectiva [mm] } \\
\hline Ptotal [mm] & 28.44 \\
\hline \% P total & 1.00 \\
\hline P cuenca & 28.44 \\
\hline Duración (horas) & 1.20 \\
\hline
\end{tabular}

Se adoptó un periodo de retorno de 3 años, aunque se entiende que para este tipo de eventos se suele analizar un periodo de 2.33 años; de igual manera, el análisis de precipitaciones máximas en 24 horas da un valor de 28.44 $\mathrm{mm}$ en la zona; este valor es el referente para determinar el hietograma de diseño. A partir de los resultados obtenidos en el cálculo del hietograma por el método del bloque alterno de la Tabla I, se obtuvo el hietograma que se muestra en la Fig. 5.

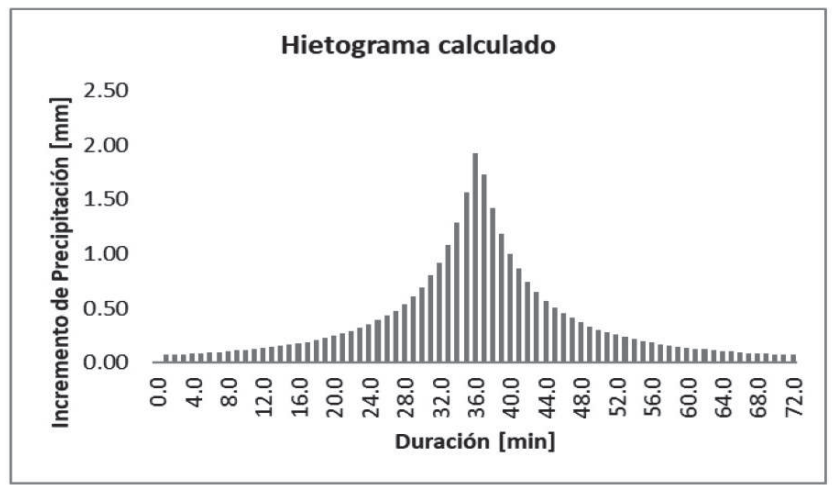

Fig. 5. Hietograma de diseño.

\section{Análisis Hidráulico}

El análisis hidráulico se determinó a partir de la metodología presentada por la EPA de acuerdo con [2] y [3], además de los parámetros estudiados e implementados de acuerdo con [10], para así dar recomendaciones sobre los valores a utilizar. Los análisis presentados en la bibliografía mencionada pueden aplicarse a los SUDS en general; para este estudio, se centran únicamente los pozos/zanjas de infiltración. Este análisis hidráulico debe estar soportado y realizado de acuerdo con la siguiente información de campo:

- Catastro de redes existentes teniendo presentes los aportes de aguas lluvias y adicionales al tramo de estudio, junto con las cotas de empate.

- Caracterización de transporte de sedimentos.

- Medición de caudales/lluvias para corroborar el correcto funcionamiento de los sistemas de drenaje sostenibles implementados.
En los trabajos de campo correspondientes a la exploración del subsuelo, se realizaron 37 perforaciones a lo largo del trazado de la tubería. La profundidad de perforación fue de 5 a $9 \mathrm{~m}$. Esta información es de vital importancia para verificar el nivel freático al que pueden estar sometidas las zanjas de infiltración, puesto que a grandes niveles puede disminuir la capacidad de funcionamiento de este sistema de drenaje complementario. La Tabla IV muestra un resumen de los trabajos de perforación realizados, así como su ubicación.

TABLA III

CÁlCUlo Del hietogRama de Diseño - Método Del BlOQue Alterno

\begin{tabular}{|c|c|c|c|c|c|c|c|}
\hline \multicolumn{8}{|c|}{ Cálculo Hietograma } \\
\hline \multirow[b]{2}{*}{ Duración } & \multirow[b]{2}{*}{ Intensidad } & \multirow{2}{*}{$\begin{array}{l}\text { Profundidad } \\
\text { acumulada }\end{array}$} & \multirow{2}{*}{$\begin{array}{l}\text { Incremento } \\
\text { profundidad }\end{array}$} & \multirow{2}{*}{\multicolumn{2}{|c|}{$\begin{array}{c}\text { Intervalo } \\
\text { de } \\
\text { tiempo }\end{array}$}} & \multicolumn{2}{|c|}{ Precipitación total } \\
\hline & & & & & & Incremento & Acumulada \\
\hline [min] & {$[\mathrm{mm} / \mathrm{hh}]$} & {$[\mathrm{mm}]$} & {$[\mathrm{mm}]$} & {$[\mathrm{mi}$} & & {$[\mathrm{mm}]$} & {$[\mathrm{mm}]$} \\
\hline 0.0 & & & & & & 0.00 & 0.00 \\
\hline 1.0 & 115.31 & 1.92 & 1.92 & 0 & 1 & 0.08 & 0.08 \\
\hline 2.0 & 109.46 & 3.65 & 1.73 & 1 & 2 & 0.08 & 0.16 \\
\hline 3.0 & 104.17 & 5.21 & 1.56 & 2 & 3 & 0.08 & 0.24 \\
\hline 4.0 & 99.37 & 6.62 & 1.42 & 3 & 4 & 0.09 & 0.33 \\
\hline 5.0 & 94.98 & 7.92 & 1.29 & 4 & 5 & 0.09 & 0.42 \\
\hline 6.0 & 90.96 & 9.10 & 1.18 & 5 & 6 & 0.10 & 0.52 \\
\hline 7.0 & 87.27 & 10.18 & 1.08 & 6 & 7 & 0.10 & 0.62 \\
\hline 8.0 & 83.86 & 11.18 & 1.00 & 7 & 8 & 0.11 & 0.73 \\
\hline 9.0 & 80.70 & 12.11 & 0.92 & 8 & 9 & 0.12 & 0.85 \\
\hline 10.0 & 77.78 & 12.96 & 0.86 & 9 & 10 & 0.12 & 0.97 \\
\hline 11.0 & 75.05 & 13.76 & 0.80 & 10 & 11 & 0.13 & 1.10 \\
\hline 12.0 & 72.51 & 14.50 & 0.74 & 11 & 12 & 0.14 & 1.24 \\
\hline 13.0 & 70.13 & 15.19 & 0.69 & 12 & 13 & 0.15 & 1.39 \\
\hline 14.0 & 67.90 & 15.84 & 0.65 & 13 & 14 & 0.16 & 1.55 \\
\hline 15.0 & 65.81 & 16.45 & 0.61 & 14 & 15 & 0.17 & 1.72 \\
\hline 16.0 & 63.84 & 17.02 & 0.57 & 15 & 16 & 0.18 & 1.90 \\
\hline 17.0 & 61.99 & 17.56 & 0.54 & 16 & 17 & 0.19 & 2.09 \\
\hline 18.0 & 60.24 & 18.07 & 0.51 & 17 & 18 & 0.21 & 2.30 \\
\hline 19.0 & 58.58 & 18.55 & 0.48 & 18 & 19 & 0.23 & 2.53 \\
\hline 20.0 & 57.01 & 19.00 & 0.45 & 19 & 20 & 0.25 & 2.78 \\
\hline 21.0 & 55.53 & 19.43 & 0.43 & 20 & 21 & 0.27 & 3.05 \\
\hline 22.0 & 54.11 & 19.84 & 0.41 & 21 & 22 & 0.29 & 3.34 \\
\hline 23.0 & 52.77 & 20.23 & 0.39 & 22 & 23 & 0.32 & 3.66 \\
\hline 24.0 & 51.49 & 20.60 & 0.37 & 23 & 24 & 0.35 & 4.01 \\
\hline 25.0 & 50.28 & 20.95 & 0.35 & 24 & 25 & 0.39 & 4.40 \\
\hline 26.0 & 49.11 & 21.28 & 0.33 & 25 & 26 & 0.43 & 4.83 \\
\hline 27.0 & 48.00 & 21.60 & 0.32 & 26 & 27 & 0.48 & 5.31 \\
\hline 28.0 & 46.94 & 21.91 & 0.30 & 27 & 28 & 0.54 & 5.85 \\
\hline 29.0 & 45.92 & 22.20 & 0.29 & 28 & 29 & 0.61 & 6.46 \\
\hline 30.0 & 44.95 & 22.48 & 0.28 & 29 & 30 & 0.69 & 7.15 \\
\hline 31.0 & 44.02 & 22.74 & 0.27 & 30 & 31 & 0.80 & 7.95 \\
\hline 32.0 & 43.12 & 23.00 & 0.26 & 31 & 32 & 0.92 & 8.87 \\
\hline 33.0 & 42.26 & 23.24 & 0.25 & 32 & 33 & 1.08 & 9.95 \\
\hline 34.0 & 41.43 & 23.48 & 0.24 & 33 & 34 & 1.29 & 11.24 \\
\hline 35.0 & 40.64 & 23.71 & 0.23 & 34 & 35 & 1.56 & 12.80 \\
\hline 36.0 & 39.87 & 23.92 & 0.22 & 35 & 36 & 1.92 & 14.72 \\
\hline 37.0 & 39.13 & 24.13 & 0.21 & 36 & 37 & 1.73 & 16.45 \\
\hline 38.0 & 38.42 & 24.33 & 0.20 & 37 & 38 & 1.42 & 17.87 \\
\hline 39.0 & 37.74 & 24.53 & 0.19 & 38 & 39 & 1.18 & 19.05 \\
\hline 40.0 & 37.07 & 24.72 & 0.19 & 39 & 40 & 1.00 & 20.05 \\
\hline 41.0 & 36.43 & 24.90 & 0.18 & 40 & 41 & 0.86 & 20.91 \\
\hline 42.0 & 35.82 & 25.07 & 0.17 & 41 & 42 & 0.74 & 21.65 \\
\hline 43.0 & 35.22 & 25.24 & 0.17 & 42 & 43 & 0.65 & 22.30 \\
\hline 44.0 & 34.64 & 25.40 & 0.16 & 43 & 44 & 0.57 & 22.87 \\
\hline 45.0 & 34.08 & 25.56 & 0.16 & 44 & 45 & 0.51 & 23.38 \\
\hline 46.0 & 33.54 & 25.71 & 0.15 & 45 & 46 & 0.45 & 23.83 \\
\hline 47.0 & 33.01 & 25.86 & 0.15 & 46 & 47 & 0.41 & 24.24 \\
\hline 48.0 & 32.50 & 26.00 & 0.14 & 47 & 48 & 0.37 & 24.61 \\
\hline 49.0 & 32.01 & 26.14 & 0.14 & 48 & 49 & 0.33 & 24.94 \\
\hline 50.0 & 31.53 & 26.27 & 0.13 & 49 & 50 & 0.30 & 25.24 \\
\hline 51.0 & 31.06 & 26.40 & 0.13 & 50 & 51 & 0.28 & 25.52 \\
\hline 52.0 & 30.61 & 26.53 & 0.13 & 51 & 52 & 0.26 & 25.78 \\
\hline 53.0 & 30.17 & 26.65 & 0.12 & 52 & 53 & 0.24 & 26.02 \\
\hline 54.0 & 29.75 & 26.77 & 0.12 & 53 & 54 & 0.22 & 26.24 \\
\hline 55.0 & 29.33 & 26.89 & 0.12 & 54 & 55 & 0.20 & 26.44 \\
\hline 56.0 & 28.93 & 27.00 & 0.11 & 55 & 56 & 0.19 & 26.63 \\
\hline 57.0 & 28.53 & 27.11 & 0.11 & 56 & 57 & 0.17 & 26.80 \\
\hline 58.0 & 28.15 & 27.21 & 0.11 & 57 & 58 & 0.16 & 26.96 \\
\hline 59.0 & 27.78 & 27.32 & 0.10 & 58 & 59 & 0.15 & 27.11 \\
\hline 60.0 & 27.42 & 27.42 & 0.10 & 59 & 60 & 0.14 & 27.25 \\
\hline 61.0 & 27.06 & 27.51 & 0.10 & 60 & 61 & 0.13 & 27.38 \\
\hline 62.0 & 26.72 & 27.61 & 0.09 & 61 & 62 & 0.13 & 27.51 \\
\hline 63.0 & 26.38 & 27.70 & 0.09 & 62 & 63 & 0.12 & 27.63 \\
\hline 64.0 & 26.06 & 27.79 & 0.09 & 63 & 64 & 0.11 & 27.74 \\
\hline 65.0 & 25.74 & 27.88 & 0.09 & 64 & 65 & 0.11 & 27.85 \\
\hline 66.0 & 25.42 & 27.97 & 0.09 & 65 & 66 & 0.10 & 27.95 \\
\hline 67.0 & 25.12 & 28.05 & 0.08 & 66 & 67 & 0.09 & 28.04 \\
\hline 68.0 & 24.82 & 28.13 & 0.08 & 67 & 68 & 0.09 & 28.13 \\
\hline 69.0 & 24.53 & 28.21 & 0.08 & 68 & 69 & 0.09 & 28.22 \\
\hline 70.0 & 24.25 & 28.29 & 0.08 & 69 & 70 & 0.08 & 28.30 \\
\hline 71.0 & 23.97 & 28.36 & 0.08 & 70 & 71 & 0.08 & 28.38 \\
\hline 72.0 & 23.70 & 28.44 & 0.07 & 71 & 72 & 0.07 & 28.45 \\
\hline
\end{tabular}


TABLA IV

UBICACIÓN Y PROFUNIDAD DE PERFORACIONES

\begin{tabular}{|c|c|c|}
\hline SONDEO & $\begin{array}{l}\text { PROFUNDIDAD } \\
\text { (m) }\end{array}$ & UBICACIÓN \\
\hline PF-1 & 6 & E 93408.49 N 107123.51 \\
\hline PF-2 & 5 & E $93376.38 \mathrm{~N} 107160.79$ \\
\hline PF-3 & 6 & E $93344.32 \mathrm{~N} 107198.09$ \\
\hline $\mathrm{PF}-4$ & 6 & E 93312.33 N 107235.51 \\
\hline PF-5 & 7 & E 93280.38 N 107272.94 \\
\hline PF-6 & 6 & E 93247.76 N 107310.21 \\
\hline $\mathrm{PF}-7$ & 7 & E $93215.17 \mathrm{~N} 107347.56$ \\
\hline PF-8 & 6 & E $93182.70 \mathrm{~N} 107374.92$ \\
\hline PF-9 & 2,5 (rechazo) & E $93150.25 \mathrm{~N} 107402.29$ \\
\hline PF-10 & 6 & E 93133.89 N 107413.82 \\
\hline PF-11 & 8 & E 93117.53 N 107425.41 \\
\hline PF-12 & 6 & E 93078.19 N 107446.63 \\
\hline PF-13 & 9 & E 93038.35 N 107468.17 \\
\hline PF-14 & 7 & E 92996.33 N 107483.77 \\
\hline PF-15 & 6 & E 92953.95 N 107499.49 \\
\hline PF-16 & 6 & E $92912.32 \mathrm{~N} 107515.95$ \\
\hline PF-17 & 8 & E $92870.25 \mathrm{~N} 107532.59$ \\
\hline PF-18 & 3 (rechazo) & E 92838.35 N 107544.52 \\
\hline PF-19 & 9,5 & E $92805.89 \mathrm{~N} 107556.67$ \\
\hline PF-20 & 7 & E 92800.77 N 107566.92 \\
\hline PF-21 & 6 & E 93398.04 N 107113.55 \\
\hline $\mathrm{PF}-22$ & 5 & E $93366.21 \mathrm{~N} 107150.88$ \\
\hline PF-23 & 7 & E $93333.72 \mathrm{~N} 107188.83$ \\
\hline PF-24 & 1,5 (rechazo) & E 93301.93 N 107225.54 \\
\hline PF-25 & 7 & E $93269.83 \mathrm{~N} 107262.72$ \\
\hline PF-26 & 6 & E $93240.84 \mathrm{~N} 107296.44$ \\
\hline PF-27 & 8 & E 93211.33 N 107330.77 \\
\hline PF-28 & 6 & E $93176.69 \mathrm{~N} 107361.37$ \\
\hline PF-29 & 7 & E $93141.49 \mathrm{~N} 107392.35$ \\
\hline PF-30 & 6 & E 93099.67 N 107418.36 \\
\hline PF-31 & 7 & E $93057.24 \mathrm{~N} 107444.79$ \\
\hline PF-32 & 6 & E $93033.29 \mathrm{~N} 107455.21$ \\
\hline PF-33 & 8 & E 93008.84 N 107465.94 \\
\hline PF-34 & 6 & E $92976.07 \mathrm{~N} 107478.62$ \\
\hline PF-35 & 7 & E 92942.76 N 107491.54 \\
\hline PF-36 & 7 & E 92896.79 N 107508.64 \\
\hline PF-37 & 8 & E 92850.52 N 107525.94 \\
\hline
\end{tabular}

En cada uno de los sondeos practicados en la zona del proyecto, se registró el nivel freático encontrado.

Para los análisis efectuados se supusieron condiciones de suelo saturado de acuerdo con la Tabla V, ya que en varios casos el nivel freático se encontró a profundidades menores de $1,5 \mathrm{~m}$. Este valor es un referente para la instalación en profundidad de las zanjas.

A partir de los resultados de las perforaciones y ensayos de laboratorio, se definieron los parámetros mostrados en la Tabla VI para cada capa de suelo

Tabla V

PROFUNDIDAD DEL NIVEL FREÁTICO ENCONTRADO

\begin{tabular}{|c|c|c|c|}
\hline \multicolumn{2}{|c|}{ COSTADO ORIENTAL } & \multicolumn{2}{c|}{ COSTADO OCCIDENTAL } \\
\hline SondeO & NF (m) & Sondeo & NF(m) \\
\hline PF-1 & 2 & PF-19 & 2,0 \\
\hline PF-2 & 2,5 & PF-20 & 6,0 \\
\hline PF-3 & 3,8 & PF-21 & 2,0 \\
\hline PF-4 & ND & PF-22 & 1,1 \\
\hline PF-5 & ND & PF-23 & 1,4 \\
\hline PF-6 & 4 & PF-24 & ND \\
\hline PF-7 & ND & PF-25 & 1,1 \\
\hline PF-8 & ND & PF-26 & 1,4 \\
\hline PF-9 & ND & PF-27 & 1,2 \\
\hline PF-10 & 3 & PF-28 & 1,5 \\
\hline PF-11 & 3,7 & PF-29 & 1,2 \\
\hline PF-12 & 2,6 & PF-30 & ND \\
\hline PF-13 & 2,5 & PF-31 & 1,0 \\
\hline PF-14 & 2,55 & PF-32 & 2,1 \\
\hline PF-15 & ND & PF-33 & 1,5 \\
\hline PF-16 & 1,1 & PF-34 & ND \\
\hline PF-17 & ND & PF-35 & 1,9 \\
\hline PF-18 & ND & PF-36 & 2,4 \\
\hline & & PF-37 & 2,3 \\
\hline
\end{tabular}

TABLA VI

Parámetros Capas De Suelo

\begin{tabular}{|c|c|c|c|c|c|c|}
\hline 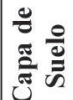 & Profundidad (m) & Descripción & $\begin{array}{c}\gamma \\
\mathrm{kN} / \mathrm{m} 3\end{array}$ & $\begin{array}{l}\varphi \\
\circ\end{array}$ & $\begin{array}{c}\mathrm{C} \\
\mathbf{k P a}\end{array}$ & $\begin{array}{l}\mathrm{Cu} \\
\mathrm{kPa}\end{array}$ \\
\hline$\overbrace{\tilde{\sigma}}^{\tilde{\sigma}}-$ & $\begin{array}{c}\text { Varía de } 0-2 \mathrm{~m} \\
\text { hasta } 0-4 \mathrm{~m}\end{array}$ & $\begin{array}{l}\text { Relleno arcillo } \\
\text { areno limoso }\end{array}$ & 20 & $23-27^{\circ}$ & - & 37 \\
\hline$\overbrace{\tilde{U}}^{\pi} \mathrm{N}$ & $\begin{array}{c}\text { Inicia de } 2 \text { a } 4 \mathrm{~m} \text { de } \\
\text { profundidad }\end{array}$ & $\begin{array}{c}\text { Arcilla o arcilla } \\
\text { limosa }\end{array}$ & 17 & 22 & 9 & 29 \\
\hline
\end{tabular}

\section{RESULTADOS}

\section{Análisis experimental}

Una vez desarrollados los componentes principales del modelo y de acuerdo con la información de referencia de la zona de este proyecto, se obtiene un hidrograma de salida en cada uno de los ductos modelados; estos hidrogramas y su respectiva reducción en el pico cuando se aplica el control LID pozos/zanjas de infiltración, serán parte esencial de los resultados obtenidos. 


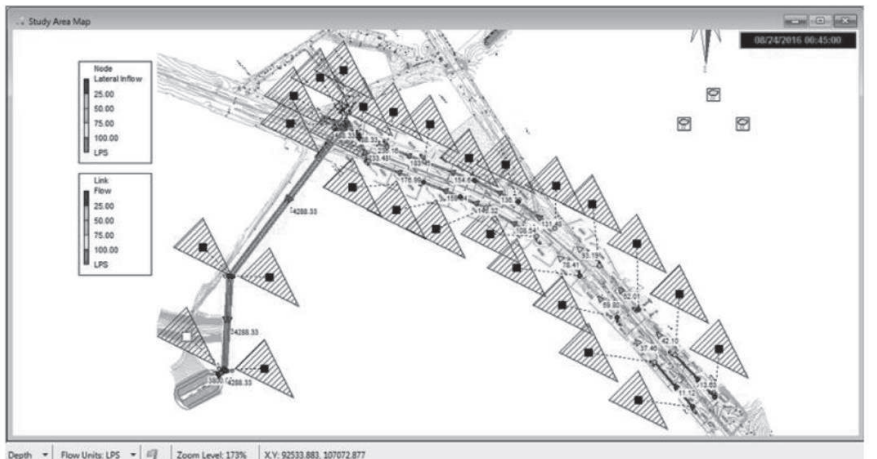

Fig. 6. Planta general del sistema de alcantarillado proyectado, modelado en EPA-SWMM.

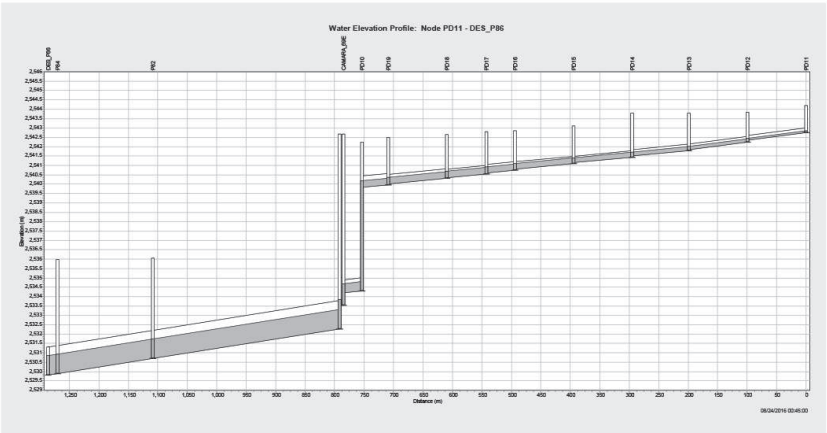

Fig. 7. Perfil del colector y la lámina de agua colector proyectado, modelado en EPA-SWMM - Flujo uniforme - Colector 1.

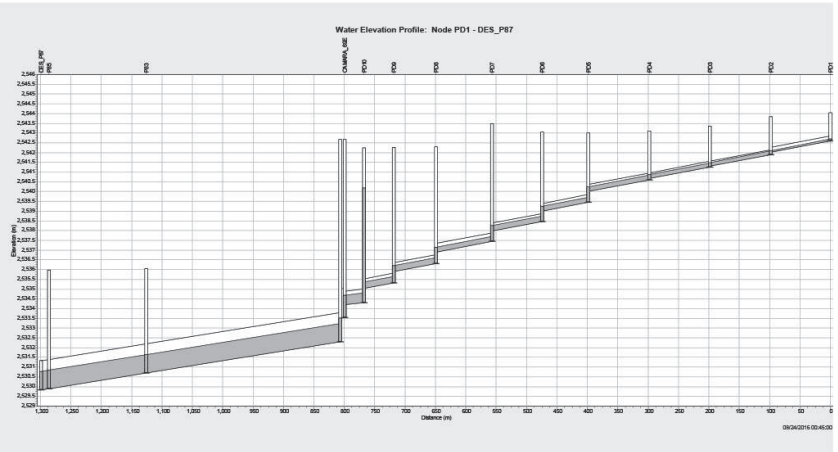

Fig. 8. Perfil del colector y la lámina de agua colector proyectado, modelado en EPA-SWMM - Flujo uniforme - Colector 2.

Dentro de este estudio se valoraron dos (2) posibles escenarios de modelación: en el primero se consideró el análisis de un colector de aguas lluvias teniendo presente una caracterización lo más completa posible del flujo, mediante el análisis y solución de las ecuaciones de Saint-Venat (característica del programa de modelación escogido). Para el segundo escenario se consideró el mismo análisis, pero fue complementado con el uso de los controles LID, también conocidos como SUDS, específicamente los pozos / zanjas de infiltración.

Este modelo puede consultarse en: https://repositorio.escuelaing.edu.co/bitstream/001/468/2/ Hoja\%20de\%20c\%c3\%a1lculo\%20SWMM.xlsm

\section{2) Aplicación de la modelación hidráulica e hidrológica}

Escenario 1 - Análisis de los colectores mediante onda dinámica:

Una vez definidos los criterios de análisis, caudales asociados y áreas aferentes de cada uno de los colectores que hacen parte del proyecto de análisis, se obtienen los hidrogramas de salida en cada uno de los nodos del sistema. Es importante aclarar que los parámetros de la cuenca fueron ajustados de acuerdo con las condiciones encontradas en los trabajos de campo realizados, y están sujetos al cambio continuo de la zona.

En la Fig. 9 y Fig. 10 se presenta el resumen de picos de los caudales obtenidos. El rango de los caudales por cada uno de los nodos es de 10 a 60 litros por segundo. El dimensionamiento de los colectores necesarios para transportar el caudal total fue realizado de acuerdo con normatividad técnica del alcantarillado de Bogotá y el reglamento técnico del sector de agua potable y saneamiento básico, RAS [11].

Escenario 2 - Análisis de los colectores mediante onda dinámica adicionando el control LID, pozos/zanjas de infiltración:

Cuando se analiza el colector implementado y los controles LID, se eligen todos los parámetros de los pozos/zanjas de infiltración, en este se tienen los valores recomendados por el manual de la EPA.

De acuerdo con el estudio de suelos en la zona del proyecto, se puede concluir la profundidad a la que se encuentra el nivel freático; esta línea freática es importante para definir si puede o no aplicarse esta técnica LID. En la Fig. 11 y Fig. 12 se presenta el resumen de los caudales obtenidos.

Para el caso del control LID se presentan la Fig. 13 y Fig. 14, donde se evidencia cuál es la reducción y el impacto de la aplicación de los sistemas de drenaje sostenibles en cada uno de los nodos.

Como punto de referencia para evaluar la reducción significativa de los caudales, se toma la descarga o cabezal de entrega final de los últimos tramos de los colectores. En las Fig. 15 y Fig. 16, Tabla VII y Tabla VIII se presentan los resultados obtenidos.

\section{Gráficos y tablas}

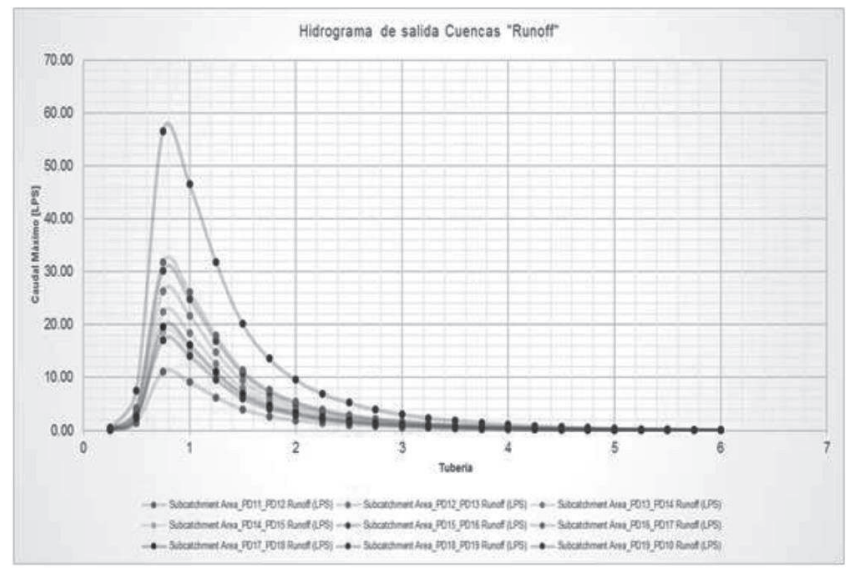

Fig. 9. Hidrogramas de salida de cada uno de los nodos (pozos) - Escenario 1 - Colector 1. 


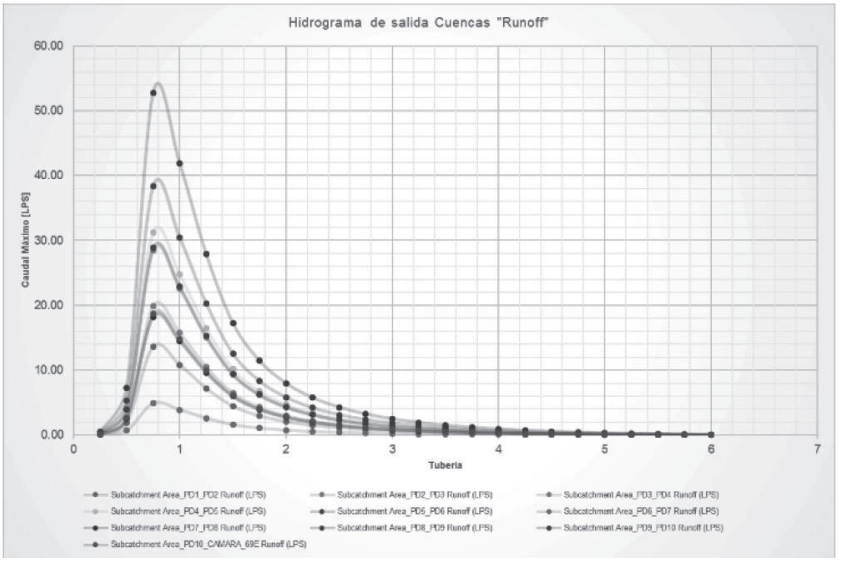

Fig. 10. Hidrogramas de salida de cada uno de los nodos (pozos) Escenario 1 - Colector 2.

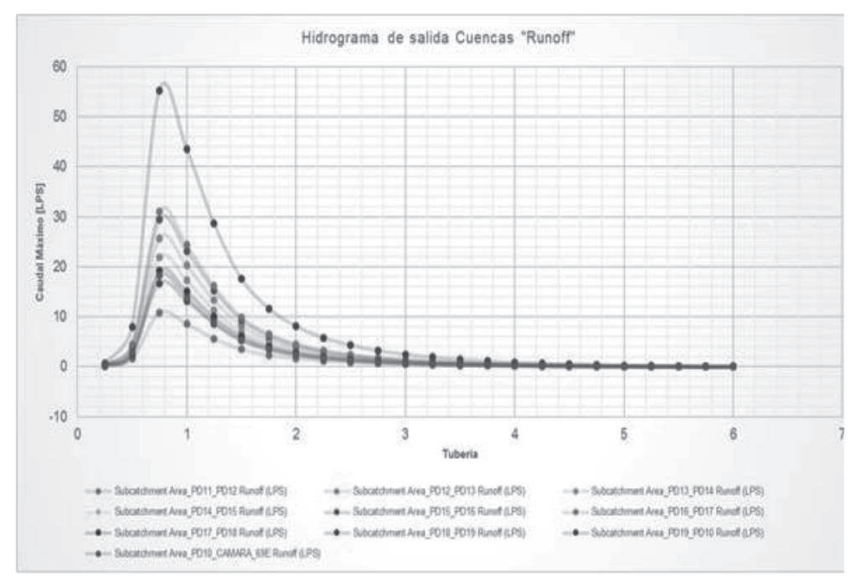

Fig. 11. Hidrogramas de salida de cada uno de los nodos (pozos) - Escenario 2 - Colector 1.

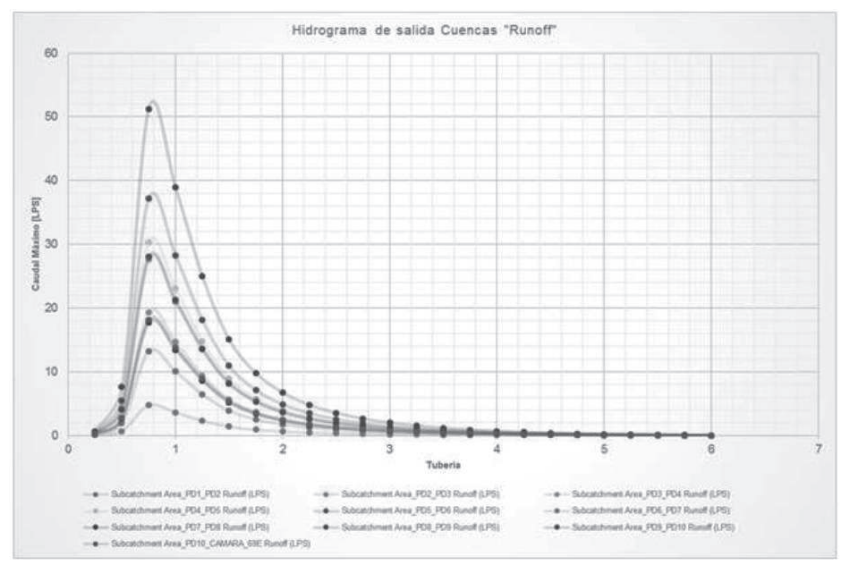

Fig. 12. Hidrogramas de salida de cada uno de los nodos (pozos) - Escenario 2 - Colector 2 .

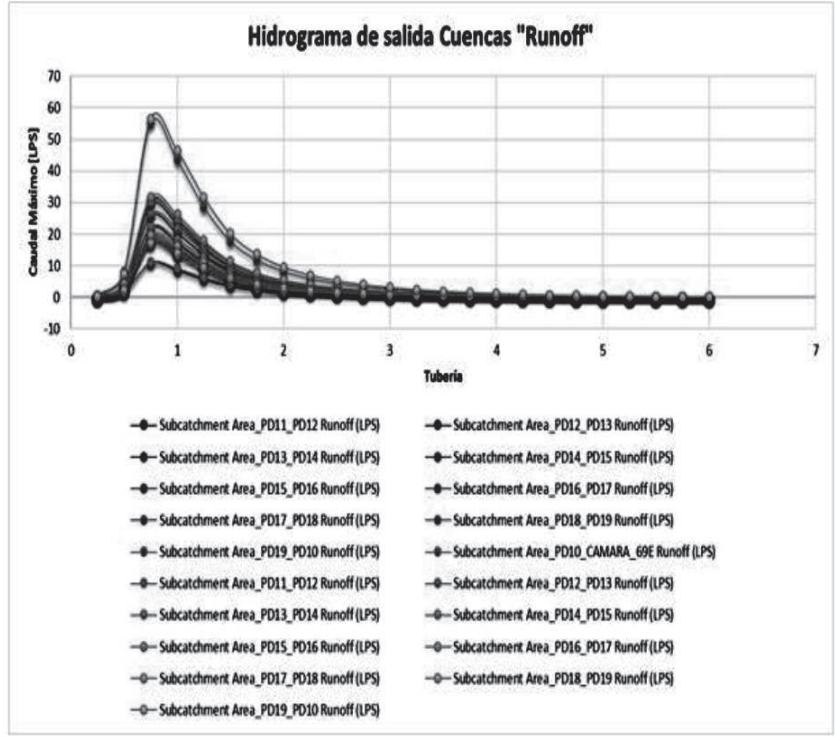

Fig. 13. Hidrogramas de salida de cada uno de los nodos (pozos) Reducción de los caudales picos - Escenario 2 - Parte 1.

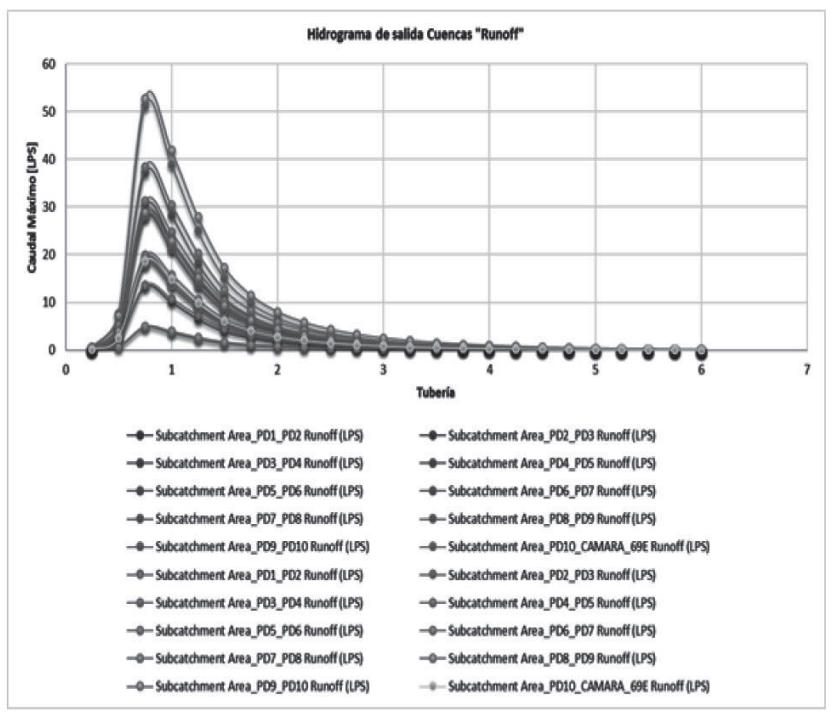

Fig. 14. Hidrogramas de salida de cada uno de los nodos (pozos) Reducción de los caudales picos - Escenario 2 - Parte 2.

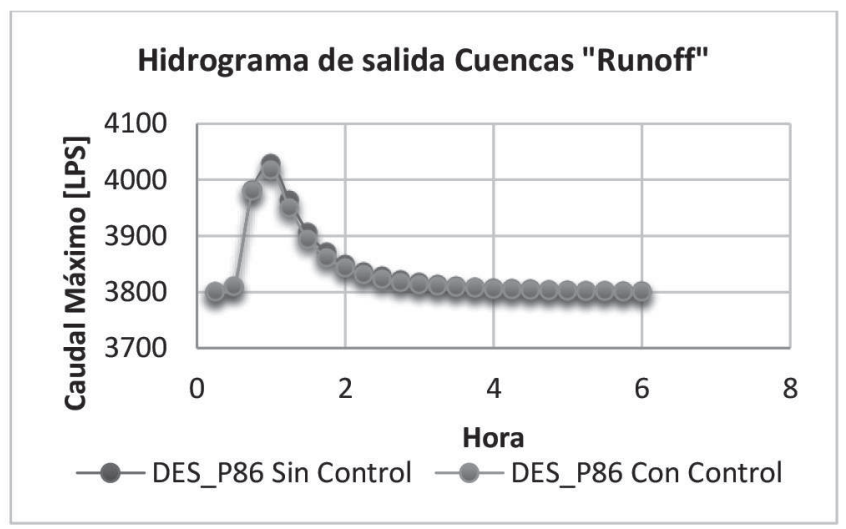

Fig. 15. Hidrogramas de salida en la descarga final - Reducción de los caudales picos - Escenario 2 - Parte 1. 


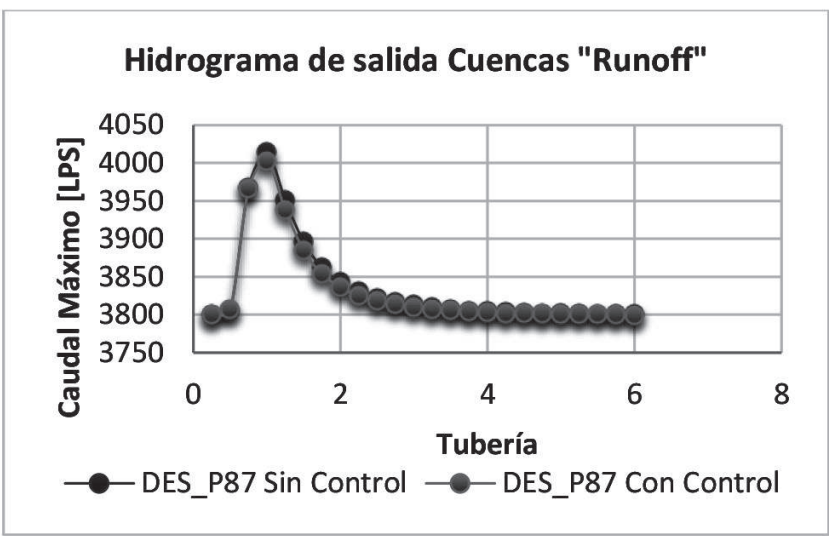

Fig. 16. Hidrogramas de salida en la descarga final - Reducción de los caudales picos - Escenario 2 - Parte 1.

TABLA VII

REDUCCIÓN DE LOS PICOS CON LA APLICACIÓN DE LOS POZOS/ZANJAS DE INFILTRACIÓN - PARTE $1-[2]$

\begin{tabular}{|c|c|c|c|}
\hline \multirow[b]{2}{*}{$\begin{array}{c}\text { Elapsed } \\
\text { Time } \\
\text { (hours) }\end{array}$} & \multicolumn{2}{|c|}{ Total Inflow (LPS) } & \multirow[b]{2}{*}{ Reducción } \\
\hline & $\begin{array}{c}\text { DES_P86 } \\
\text { Sin } \\
\text { Control } \\
\end{array}$ & $\begin{array}{c}\text { DES_P86 } \\
\text { Con Control }\end{array}$ & \\
\hline 0.25 & 3800.0935 & 3800.6025 & -0.509 \\
\hline 0.5 & 3808.5923 & 3810.0569 & -1.4646 \\
\hline 0.75 & 3981.592 & 3980.2366 & 1.3554 \\
\hline 1 & 4029.0815 & 4017.3699 & 11.7116 \\
\hline 1.25 & 3963.4856 & 3950.1238 & 13.3618 \\
\hline 1.5 & 3906.1184 & 3893.7703 & 12.3481 \\
\hline 1.75 & 3871.0078 & 3861.4644 & 9.5434 \\
\hline 2 & 3849.4741 & 3843.2988 & 6.1753 \\
\hline 2.25 & 3836.0657 & 3831.0261 & 5.0396 \\
\hline 2.5 & 3828.0146 & 3823.0532 & 4.9614 \\
\hline 2.75 & 3821.835 & 3817.0027 & 4.8323 \\
\hline 3 & 3816.7517 & 3813.4548 & 3.2969 \\
\hline 3.25 & 3813.209 & 3811.1421 & 2.0669 \\
\hline 3.5 & 3810.4453 & 3808.9373 & 1.508 \\
\hline 3.75 & 3808.0627 & 3806.9751 & 1.0876 \\
\hline 4 & 3806.8345 & 3805.0667 & 1.7678 \\
\hline 4.25 & 3805.6799 & 3804.4771 & 1.2028 \\
\hline 4.5 & 3804.7183 & 3803.4292 & 1.2891 \\
\hline 4.75 & 3803.7295 & 3802.6914 & 1.0381 \\
\hline 5 & 3802.8289 & 3802.0852 & 0.7437 \\
\hline 5.25 & 3802.218 & 3801.5754 & 0.6426 \\
\hline 5.5 & 3801.6982 & 3801.115 & 0.5832 \\
\hline 5.75 & 3801.2217 & 3800.8538 & 0.3679 \\
\hline 6 & 3800.9224 & 3800.6035 & 0.3189 \\
\hline & & $Q=$ & 83.2688 \\
\hline
\end{tabular}

De acuerdo con los resultados obtenidos en el análisis descrito previamente, la correcta aplicación de los controles LID, como el pozo/zanja de infiltración, permite aminorar los picos de las tormentas o eventos máximos que se presenten en determinadas zonas. Es importante aclarar que bajo condiciones ideales, estos se comportan de manera teóricamente correcta, garantizando esta disminución.

Los resultados obtenidos como objeto de este estudio pretenden resaltar la importancia del uso de alternativas sostenibles de drenaje urbano para disminuir los aportes de los colectores y evitar fenómenos como inundaciones. También pueden usarse para mejorar la calidad de agua del agua lluvia.
TABLA VIII

REDUCCIÓN DE LOS PICOS CON LA APLICACIÓN DE LOS POZOS/ZANJAS DE INFILTRACIÓN - PARTE 2

\begin{tabular}{|c|c|c|c|}
\hline \multirow[b]{2}{*}{$\begin{array}{l}\text { Elapsed } \\
\text { Time } \\
\text { (hours) }\end{array}$} & \multicolumn{2}{|c|}{ Total Inflow (LPS) } & \multirow[b]{2}{*}{ Reducción } \\
\hline & $\begin{array}{c}\text { DES_P87 } \\
\text { Sin } \\
\text { Control }\end{array}$ & $\begin{array}{c}\text { DES_P87 } \\
\text { Con Control }\end{array}$ & \\
\hline 0.25 & 3800.0051 & 3800.0835 & -0.0784 \\
\hline 0.5 & 3805.563 & 3806.8662 & -1.3032 \\
\hline 0.75 & 3967.5989 & 3966.3247 & 1.2742 \\
\hline 1 & 4014.0154 & 4002.2456 & 11.7698 \\
\hline 1.25 & 3950.9658 & 3938.1116 & 12.8542 \\
\hline 1.5 & 3895.9531 & 3884.9663 & 10.9868 \\
\hline 1.75 & 3862.4409 & 3853.8845 & 8.5564 \\
\hline 2 & 3842.925 & 3836.5249 & 6.4001 \\
\hline 2.25 & 3830.7461 & 3825.0105 & 5.7356 \\
\hline 2.5 & 3821.75 & 3818.3696 & 3.3804 \\
\hline 2.75 & 3816.6826 & 3813.5374 & 3.1452 \\
\hline 3 & 3813.0652 & 3809.8774 & 3.1878 \\
\hline 3.25 & 3809.9075 & 3806.958 & 2.9495 \\
\hline 3.5 & 3807.0952 & 3805.9314 & 1.1638 \\
\hline 3.75 & 3804.7813 & 3804.3782 & 0.4031 \\
\hline 4 & 3804.5713 & 3802.8997 & 1.6716 \\
\hline 4.25 & 3803.4526 & 3801.5808 & 1.8718 \\
\hline 4.5 & 3802.2656 & 3802.1213 & 0.1443 \\
\hline 4.75 & 3801.1172 & 3801.4451 & -0.3279 \\
\hline 5 & 3801.7556 & 3800.4998 & 1.2558 \\
\hline 5.25 & 3801.1526 & 3800.894 & 0.2586 \\
\hline 5.5 & 3800.2666 & 3800.6914 & -0.4248 \\
\hline 5.75 & 3800.7268 & 3800.0159 & 0.7109 \\
\hline 6 & 3800.5171 & 3799.3125 & 1.2046 \\
\hline & & $\mathbf{Q}=$ & 76.7902 \\
\hline
\end{tabular}

\section{CONCLuSiones}

\section{A. Generales}

El uso de nuevas alternativas de drenaje urbano como complemento de los sistemas de drenajes existentes, permite mejorar de manera significativa la eficiencia de desagües de aguas lluvias en una ciudad. Es importante que los sistemas sean planteados, diseñados, construidos y mantenidos para que su propósito sea efectivo, mitigando y realizando un control de los efectos adversos que la escorrentía provoca al medio ambiente.

En Colombia, el uso de SUDS como complemento de los sistemas de drenaje convencionales empieza a tener un auge importante, pues se evidencia la necesidad de usar nuevas alternativas para disminuir los aportes de escorrentía en redes de aguas lluvias proyectadas, y que al mismo tiempo sean amigables con el ambiente, permitiendo la menor afectación. Algunos de los beneficios que se obtienen con estos sistemas son:

- Aminorar los caudales pico disminuyendo el riesgo de que se presenten fenómenos de inundación.

- Reducir volumen y frecuencia de escorrentías desde áreas urbanizadas hacia cauces naturales o redes de alcantarillado, para reproducir el drenaje natural y reducir el riesgo de inundación.

- Mejorar la calidad del agua procedente de las escorrentías, 
eliminando los contaminantes procedentes de fuentes difusas.

- Mejorar el paisaje urbano y ofrecer un mejor servicio al ciudadano.

- Reducir el número de descargas directas a la red.

- Minimizar la afección al régimen de funcionamiento de los cauces naturales receptores de las redes de alcantarillado pluvial.

- Mejorar el flujo subterráneo hacia los cursos naturales mediante infiltración.

\section{B. Especificas}

- Los trabajos de campo deberán ser muy cuidadosos, sobre todo cuando se implementen sistemas SUDS, puesto que las condiciones del suelo van a regir la instalación de estos elementos.

- Los caudales fueron reducidos en un rango de 84 y 76 litros por segundo, es decir, este volumen no fue aportado ni transportado por la red proyectada, permitiendo que fuese una red óptima.

- La modelación hidráulica de las redes de alcantarillado pluvial permite analizar un proyecto con parámetros cada vez más reales, a partir de valores medibles (en este caso, precipitaciones).

- Es importante revisar la información que es cargada a EPA-SWMM, para evitar aseveraciones incorrectas.

- El resultado del estudio arroja que los picos máximos producidos por los hietogramas de diseño pueden ser aminorados mediante el uso de sistemas SUDS, sin embargo, para corroborar esta hipótesis es necesario que se realice una medición continua de los caudales o parámetros hidrológicos adoptados por cada colector, una vez se conocen los aportes y el tramo en estudio.

- En la ejecución de proyectos no es usual el uso de información reciente en el análisis hidrológico e hidráulico, es decir, no se realiza una investigación de calidad, esto da como resultado un diseño que asume muchos parámetros, pudiéndose sobredimensionar los elementos diseñados y en algunos casos, generando grandes gastos en la administración municipal o de ciudad.

\section{Estudios e información necesaria}

Un aspecto definitivo para realizar un diseño con el que se pretenda incursionar en la aplicación de SUDS, son los estudios de la zona de influencia, información como topografía, uso del suelo, hidráulicos e hidrológicos, tipo de suelo, niveles freáticos y otros estudios técnicos deberán analizarse antes de la aplicación de estos sistemas, pues es importante aclarar que de acuerdo con las características propias del lugar se podrá o no implementar.

Asimismo, es necesario apoyarse en estudios técnicos y pruebas de laboratorio que complementen el trabajo de diseño junto con la construcción en distintos tipos de suelos, analizando su comportamiento hidráulico frente a la zonificación colombiana.

\section{Costos en la red de alcantarillado pluvial}

El uso de SUDS en combinación con los sistemas de drenaje urbano convencionales, permite disminuir el caudal aportado en todo el sistema, lo cual se refleja en una disminución de diámetros y de pendientes en la red planteada y posibilitando. Esto a su vez, conduce a que no se construyan redes con excavaciones de gran profundidad, logrando reducir los costos de todo el sistema proyectado.

\section{E. Aplicación en la normatividad colombiana}

En Colombia, es necesario que las entidades encargadas del control de escorrentías en las ciudades y con el apoyo de los estudios técnicos requeridos, se encarguen de divulgar los beneficios que los sistemas SUDS traen consigo. Además, es importante que el proceso de implementación de estos sistemas en los diseños de cada ciudad tenga presente las siguientes fases:

- Concienciación (ciudadanía, empresas públicas y privadas, entre otros).

- Elaboración de manuales de diseño y construcción.

- Normatividad y legislación.

- Monitorización de la aplicación de estos sistemas que se van construyendo.

- Investigación de nuevas técnicas, materiales, procesos constructivos, entre otros, con el fin de mejorar los sistemas estudiados.

- Actualización continua de los conocimientos adquiridos a través de manuales y de la normativa.

\section{ACLARACIONES}

La información para la descripción y análisis de cada uno de los escenarios que se ofrecen en este artículo, fue tomada del trabajo de grado "Control de escorrentías urbanas mediante pozos de infiltración: Aplicación práctica a un tramo del Colector de aguas lluvias de la localidad de Fontibón, Bogotá, D.C.”, y fueron usados con propósitos netamente educativos, con el fin de dar pie a nuevos estudios relacionados con los sistemas de drenaje sostenible y mejorar la manera en la que se analizan los proyectos de drenaje de aguas lluvias en la ciudad de Bogotá, Colombia.

\section{REFERENCIAS}

[1] Banco MundialColombia, Gestion del Riesgo. «http://gestiondelriesgo. gov.co,» 2012. [En línea]. Available: http://gestiondelriesgo.gov.co/ sigpad/archivos/GESTIONDELRIESGOWEB.pdf. Recuperado el 22 de abril de 2018.

[2] Us Environmental Protection Agency, Storm Water Management Model Reference Manual, vol. I - Hidrology, Us Environmental Protection Agency, 2016.

[3] US Environmental Protection Agency, Storm Water Management Model Reference Manual, vol. III - Water Quality, US Environmental Protection Agency, 2016.

[4] Rodriguez, J., Rodríguez, J., Gómez, E. y Castro, D., "Sistemas urbanos de drenaje sostenible, SUDS", 2009. [En línea]. Available: http://www.caminospaisvasco.com/Profesion/Publicaciones\%20 $\mathrm{de} \% 20$ nuestros $\% 20$ colegiados/suds. 
[5] Secretaría Distrital de Ambiente - Subdirección de Ecourbanismo y Gestión Ambiental, «http://ambientebogota.gov.co/,» 2011. [En línea]. Available: http://ambientebogota.gov.co/documents/10157/73754/ Sistema+Urbanos+de+Drenaje+Sostenible.

[6] Sánchez, J. R., "Nuevas tendencias en la gestión de drenaje pluvial en una cuenca urbana", España: Universidad de la Rioja, 2012.

[7] Construction Industry Research and Information Association, CIRIA C523 (2001). Sustainable urban drainage systems, best practice manual for England, Scotland, Wales and Northern Ireland, Londres, RU: Construction Industry Research and Information Association, 2001, p. 131.

[8] Ministerio de Vivienda y Urbanismo. Dirección de Investigaciones Científicas y Tecnológicas. Técnicas alternativas para soluciones de aguas lluvias en sectores urbanos. Guía de diseño, Santiago, Chile, 1996, p. 670.

[9] Chow, V. T., Maidment, D. R. y Mays, L. W., Hidrología Aplicada McGraw-Hill Interamericana, S.A., 1994.

[10] Cano, O. M., "Low impacy development (LID) opportunities in the district of lamúd, Perú: a study of hydrology, cost and maintenance of appropriate stormwater best management practices (BMPs)", Michigan Technological University, Michigan, EEUU, 2015.

[11] Ministerio de Vivienda, Ciudad y Territorio, «www.minvivienda.gov. co,» 01 Diciembre 2000. [En línea]. Available: http://www.minvivienda.gov.co/Documents/ViceministerioAgua/TITULO_D.pdf. [Último acceso: 2016].

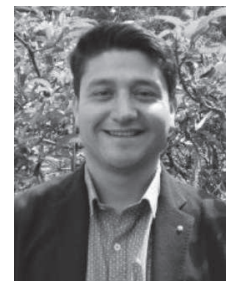

Elkin Cubides. Ingeniero Civil de la Escuela Colombiana de Ingeniería. Magister en Ingeniería Civil con énfasis en Recursos Hidráulicos y Medio Ambiente de la Escuela Colombiana de Ingeniería. Especialista en Gerencia de Proyectos de Construcción e infraestructura del Colegio Mayor de Nuestra Señora del Rosario. Actualmente se desempeña como consultor privado. Experto en hidráulica e hidrología.

ORCID: https://orcid.org/0000-0001-5342-7663.

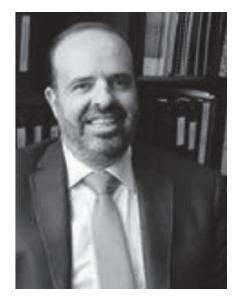

Germán Santos. Ingeniero Civil de la Escuela Colombiana de Ingeniería. Master of Science en Recursos Hidráulicos y Ph.D. en Engineering Science and Mechanics de Virginia Tech. Actualmente se desempeña como profesor titular de la Escuela Colombiana de Ingeniería. Experto en hidráulica e hidrología computacional.

ORCID: https://orcid.org/0000-0002-7488-879X. 